\title{
EL APRENDIZAJE HISTOLÓGICO INICIAL DE SANTIAGO RAMÓN Y CAJAL: NOTAS ACERCA DE SUS INVESTIGACIONES SOBRE LA INFLAMACIÓN
}

\author{
Alfredo Rodríguez Quiroga
}

Residencia de Estudiantes, CSIC

\section{RESUMEN}

Cajal adquirió la formación histológica que sirvió de punto de partida a su genial obra sobre la estructura del sistema nervioso durante la década comprendida entre mediados de 1877 y finales de 1887. De modo más exacto, su aprendizaje histológico inicial comenzó a fraguarse precisamente desde que cursó el doctorado con Aureliano Maestre de San Juan y se graduó con su discurso sobre la Patogenia de la inflamación, tema al que dedicaría tres años más tarde su primera publicación. Esta primera etapa de la trayectoria científica de Cajal ha sido, generalmente, obviada, toda vez que su dedicación de modo continuado a la histología no se produjo hasta el otoño de 1885 , siendo catedrático de anatomía en Valencia. En este sentido, la bibliografía de carácter más o menos panegírico que se ha ocupado de su figura ha contribuido en gran medida a minimizar en la mayoría de los casos este decisivo período de su carrera científica. En este trabajo pretendemos, pues, contribuir a paliar esta laguna de la historiografía cajaliana, recuperando en primera instancia el mencionado ejercicio con el cual Cajal se graduó como doctor en 1877 y, subsiguientemente, los primeros ensayos micrográficos que, a raíz de la redacción de su memoria emprendió a lo largo de los tres años siguientes y que se tradujeron en su primera monografía publicada.

PALABRAS CLAVE: Cajal, inflamación. doctorado, histología, aprendizaje.

\section{SUMMARY}

Cajal attained the histological training which was the starting point for his brilliant work on the structure of the nervous system during the decade from the middle of 1877 to the end of 1887 . More exactly, his initial histological training began to develop blossom once he did his $\mathrm{Ph}$. D. with Aureliano Maestre de San Juan and he graduated with his thesis on the Pathogenesis of inflamation, which would be the subject of his first publication three years later. This first stage of Cajal's scientific career has been, on the whole, forgotten, as his full time work on histology did not take place till the Autumn of 1885, when he was Professor of Anatomy at Valencia. Therefore, the bibliography of a more or less panegyrical nature on this figure has led largely to the underrating, in most cases, of this decisive period in his scientific career. In study we aim, therefore, to attempt to fill this gap in «Cajalian» historiography, by firstly looking at the above-mentioned Doctorate thesis of 1877, and subsequently the first micrographical essays wich he worked on for the following three years, as a resultt of the writing of his thesis and which became his first published monograph.

KEY WORDS: Cajal, inflamation, doctorate, histology, training. 


\section{INTRODUCCIÓN}

Tras permanecer casi dos años en Cuba como médico militar y una vez repatriado con la salud gravemente quebrantada, a su regreso a España en junio de 1875, Cajal, carente de afición para dedicarse al ejercicio clínico de la profesión, comenzó su preparación para concurrir a las pertinentes oposiciones a cátedras de anatomía. En noviembre de ese mismo año, fue nombrado ayudante interino de Anatomía por la «Comisión Mixta de Estudios Médicos» de la que, como es sabido, dependía la Escuela de Medicina de Zaragoza, y cuyo ambiente limitó en un principio el horizonte científico de Cajal. En mayo del año siguiente obtuvo por oposición una plaza de «practicante de primera clase» en el Hospital de Nuestra Sra. de Gracia. Finalmente, en abril de 1877 fue designado profesor auxiliar interino de la recién creada Facultad de Medicina oficial de Zaragoza, antes, como ha quedado apuntado, con categoría de Escuela.

Sin embargo, para aspirar al profesorado universitario era obligatorio obtener el título de doctor, que entonces sólo podía cursarse en la Universidad Central de Madrid, a pesar de lo cual Cajal permaneció en Zaragoza en calidad de alumno libre. En junio de 1877 se trasladó a Madrid para presentarse a los exámenes de las tres asignaturas de que constaban entonces los estudios de doctorado: «Historia de la Medicina», «Análisis químico aplicado a las ciencias médicas» - materias en las que sufrió una fuerte decepción- e «Histología normal y patológica», disciplina de la que era catedrático titular Aureliano Maestre de San Juan'1, con quien Cajal inició, como más adelante veremos, una profunda relación que condicionó su posterior trayectoria científica. De hecho, el propio Maestre apadrinó su ejercicio de doctorado, pues, según disponía la legislación vigente: «El que aspire al grado de doctor escribirá sobre el asunto que prefiera entre los comprendidos en (según se relacionan), un discurso, cuya lectura no dure más de media hora ni menos de veinticinco minutos, tomándose para hacer este trabajo, el tiempo que tenga por conveniente ... el ejercicio de doctorado consistirá en la lectura del discurso ... y en las observaciones que sobre él harán al graduando, por espacio de un cuarto de hora, cada uno de los tres jueces que designe el pre-

1 GARCÍA DEL REAL, E. (1935), «Dos precursores de Ramón y Cajal: Maestre de San Juan y López García», Trabajos de la Cátedra de Historia Crítica de la Medicina, 4, pp. 479-494; LÓPEZ PIÑERO, J. M. (1973), «Maestre de San Juan, cabeza de la histología española anterior a Cajal», Noticias Médicas (25 febrero), pp. 9-11; LÓPEZ PIÑERO, J. M., TERRADA, M. L., BujosA, F. (1979), Clásicos españoles de la anatomía patológica anteriores a Cajal, Valencia, Cátedra de Historia de la Medicina; LÓPEZ PIÑERO, J. M. (1983), «Maestre de San Juan, Aureliano», en: LÓPEZ PIÑERO, J. M.; GLICK, T. F.; NAVARRO Brotóns, V.; PORTEla MARco, E. (eds.), Diccionario histórico de la ciencia moderna en España, Barcelona, Península, vol. II, pp. 11-13; MARCO CUÉLlAR, R. (1966), La morfología microscópica normal y patológica en la medicina española del siglo XIX anterior a Cajal, Valencia, tesis doctoral; Olagüe de Ros, G., et al. (1985), «La obra histológica de Aureliano Maestre de San Juan y Muñoz (18281890)», Histología Médica, 1, pp. 143-146; ViñAls y TORRERO, F. (1885), Apuntes biográficos del Dr. Aureliano Maestre de San Juan, Madrid. F. García Herrero. 
sidente» ${ }^{2}$. El discurso de Cajal versó sobre la Patogenia de la inflamación y está fechado el 26 de junio de $1877^{3}$, no obstante su lectura y defensa tuvo lugar el 3 de julio siguiente, siendo finalmente calificado de aprobado, según consta en el acta correspondiente ${ }^{4}$. Ejerció de presidente Julián Calleja y completaron el tribunal, además del propio Maestre, Teodoro Yáñez, catedrático de Medicina Legal, y Carlos Quijano, titular de Higiene. Se trata, sin embargo, de un texto cajaliano que ha sido generalmente discriminado en la historiografía médica, quizá porque, como acertadamente apunta José M. López Piñero en su biografía del gran histólogo ${ }^{5}$, el propio Cajal no hace referencia explícita a él en sus Recuerdos ${ }^{6}$. Por tanto, el objetivo aquí propuesto no es otro que la recuperación de dicho texto que constituyó al mismo tiempo el germen de su primera publicación científica tres años más tarde, en 1880, y, en última instancia, el punto de partida de su formación histológica.

\section{EL PROCESO FLEGMÁSICO: DEFINICIÓN Y PATOGENIA}

El tema de la inflamación, definido por Cajal como «uno de los actos más generales y espontáneos de la organización enferma» ${ }^{7}$, era, ciertamente, uno de los puntos sometidos en aquel momento a la controversia médica cuya aclaración entrañaba más trascendencia para la exacta comprensión de la génesis de muchísimas dolencias. Es preciso señalar que, con anterioridad, en su época de estudiante, la cuestión había motivado un caluroso enfrentamiento entre Cajal, profundamente imbuido de las teorías celularistas de Virchow — cuya Die cellular Pathologie ${ }^{8}$ había sido vertida recientemente al castellano (1868) - y Genaro Casas, decano y encargado de la enseñanza de la Patología Médica en la Escuela de Zaragoza, ardiente defensor de las tradicionales ideas vitalistas $^{10}$.

2 Oyuelos PÉReZ, R. (1895), Legislación de Medicina. Estudio del Derecho vigente (administrativo, civil, penal y procesal) y jurisprudencia del Tribunal Supremo, referente a la Facultad de Medicina, Madrid, Impr. de M. Rojas, vol. I, pp. 15-16.

3 RAMÓn y CAJAL, S. (1877), Patogenia de la inflamación. Discurso para los ejercicios del grado de Doctor... Madrid, Universidad Central, Facultad de Medicina, legajo 25, ${ }^{\circ} 29$.

4 Expedientes administrativos de grandes españoles, II. Santiago Ramón y Cajal, Madrid, Ministerio de Educación y Ciencia, 1978, vol. I, pp. 107-108; 151-152; 154-155.

5 LÓPEZ PIÑERO, J. M. (1995), Ramón y Cajal, Barcelona, Salvat.

6 RAMÓN Y CAJAL, S. (1923), Recuerdos de mi vida, Madrid, J. Pueyo.

7 RAMÓN Y CAJAL (1877).

8 VIRCHOW R. (1858), Die cellular pathologie in ihrer begrundung auf physiologische und pathologische gewebelehre. Zwanzig vorlesungen gehalten während der monate februar, märz und april 1858 im Pathologischen institute zu Berlin, Berlín, A. Hirschwald.

9 VirChOW, R. (1868), La Patología celular fundada en el estudio fisiológico y patológico de los tejidos... (Traducida de la segunda edición alemana por Pablo Picard, vertida al español y anotada por Juan Giné y Bartolomé Robert), Madrid, Imprenta Española.

10 RAMÓN Y CAJAL (1923). 
En su discurso de doctorado sobre el proceso inflamatorio considerado bajo su aspecto patogénico, Cajal se limitó básicamente a realizar una exposición y emitir un breve juicio crítico acerca de «las más notables y recientes investigaciones y doctrinas encaminadas a esclarecer el mecanismo de este complejo estado patológico» ${ }^{11}$. Se trataba, en suma, de una memoria de carácter meramente teórico, vertebrada conforme al esquema trazado por Eduardo García Solá en su Tratado de Patología general y Anatomía patológica (1874) ${ }^{12}$. Cajal comenzó, pues, por recoger, siguiendo un orden no siempre rigurosamente cronológico, las principales teorías e interpretaciones que los patólogos habían enunciado para tratar de explicar racionalmente la génesis del proceso inflamatorio. Así, partiendo de la definición sintomatológica que de este fenómeno morboso realizara Celso, caracterizándolo por los cuatro síntomas cardinales: rubor, tumor, calor y dolor: «Notoe vero inflamationis sunt quatuor, rubor et tumor, cum calore et dolore» ${ }^{13}$, a la que posteriormente se añadió el functio laesa (trastorno funcional), Cajal describió, en primer término, las primitivas teorías vasculares que, ignorando la textura íntima de los órganos y basadas en los caracteres aparentes, consideraban la modificación del estado circulatorio como el fenómeno más importante en la inflamación.

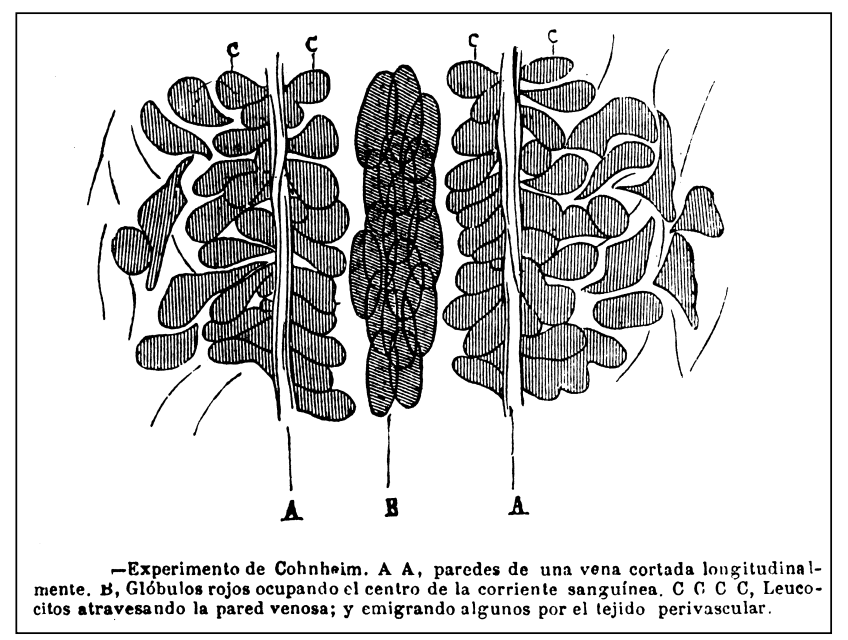

Experimento de Cohnheim (1867). (Figura extraida del Tratado de Patología General y Anatomía Patológica de Eduardo García Solá, 1874).

1 RAMÓN Y CAJAL (1877)

12 García SolÁ, E. (1874), Tratado de Patología general y Anatomía patológica, Madrid, Moya y Plaza.

13 Celso, A. C. (1823), De re medica libri octo, París, J. B. Baillière, Libro 311, cap. XI, sec. VI, pág. 130. 


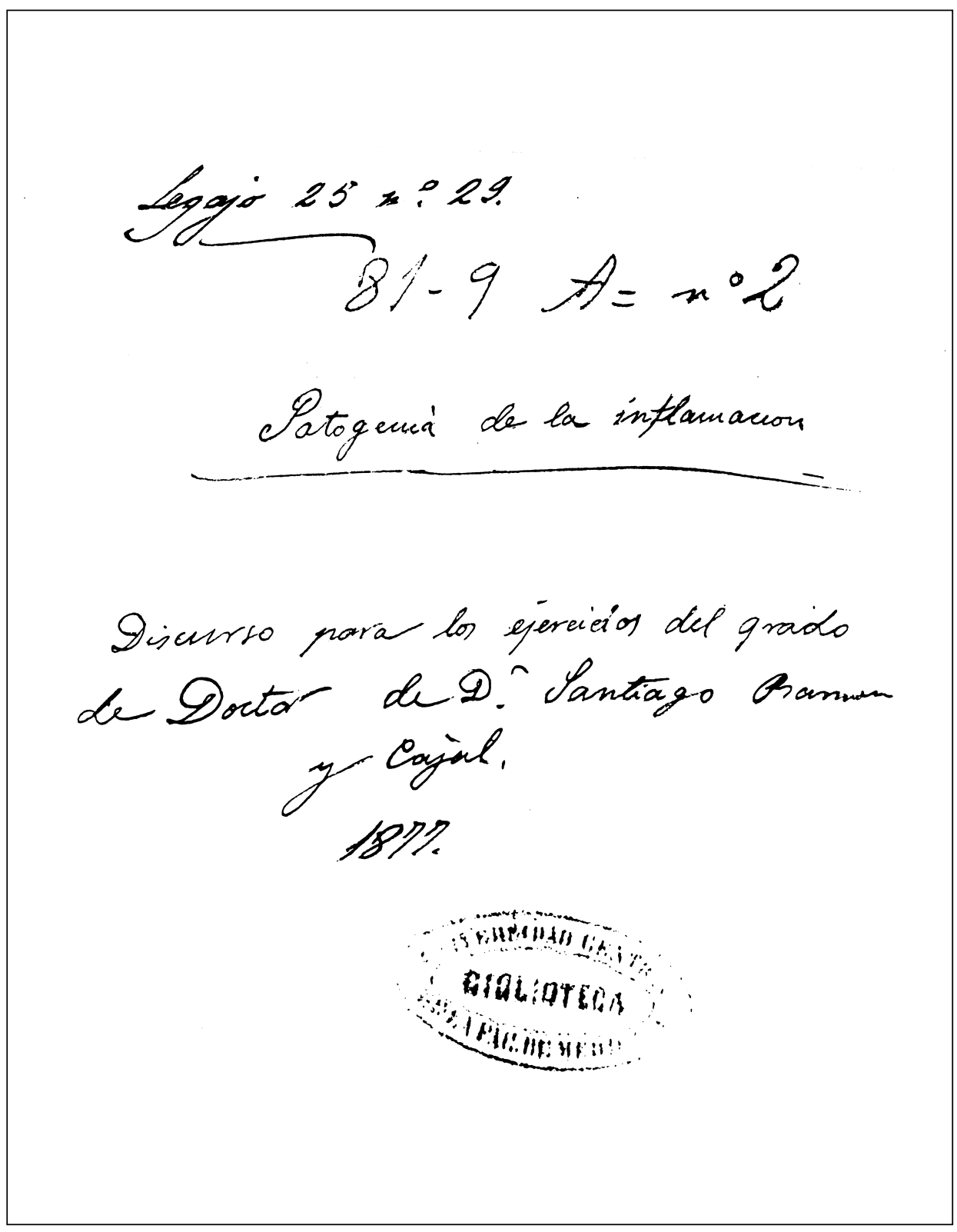

Portada del discurso de doctorado y páginas correspondientes al texto manuscrito del ejercicio de doctorado de Cajal sobre la Patogenia de la Inflamación (1877). 


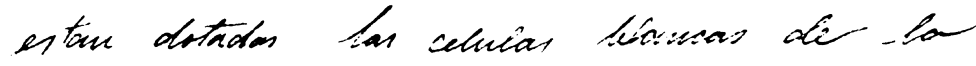

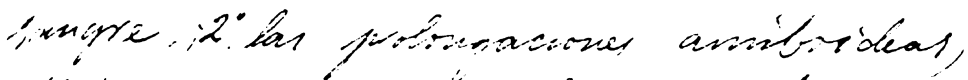

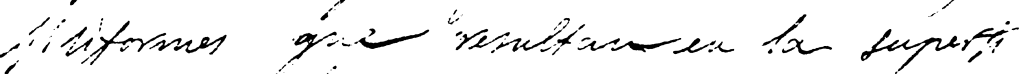

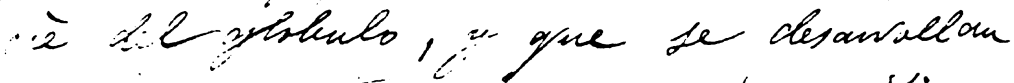
1:- el momenti engue te shmoviliza

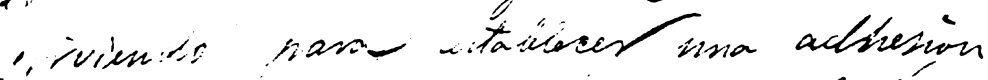
itima cille at stamati is ef plobicio. 3. f. ivenivi de los vestricte lencontos que fincien -isiden a entrivaririesy give

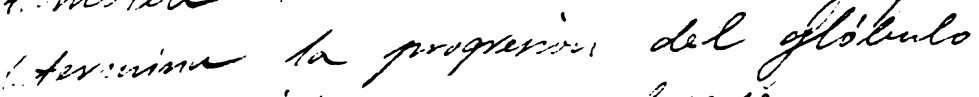

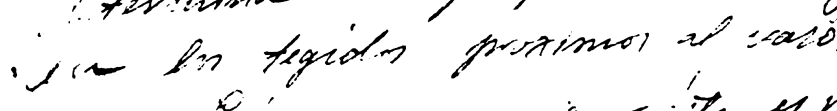

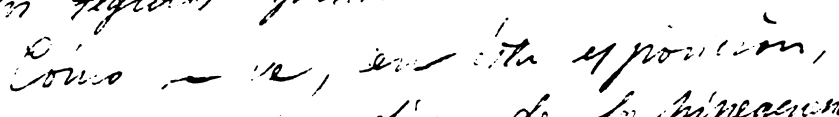

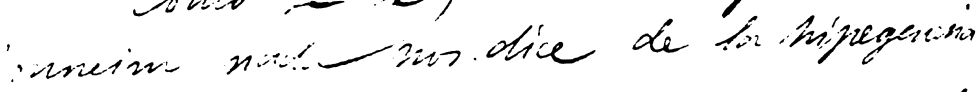

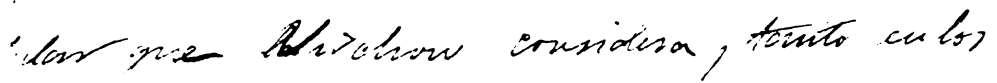

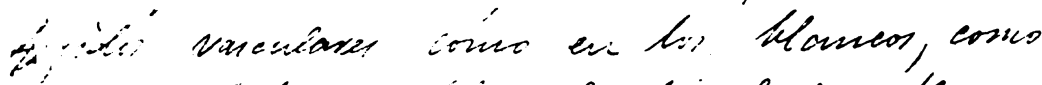
i yinto de jaxtra de la afeceror thequa.

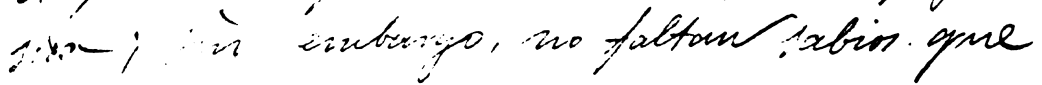

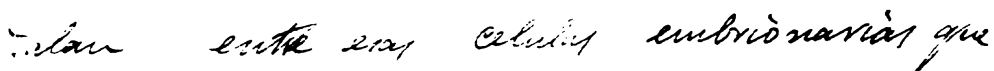

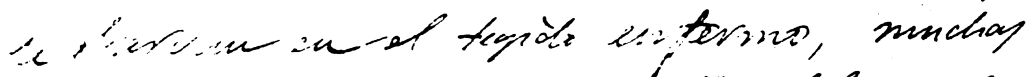

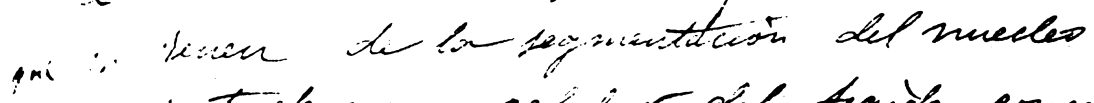

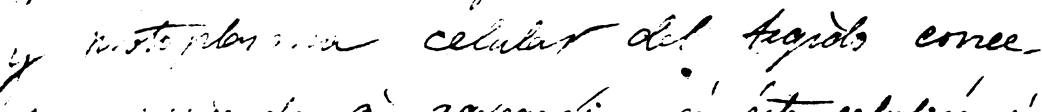

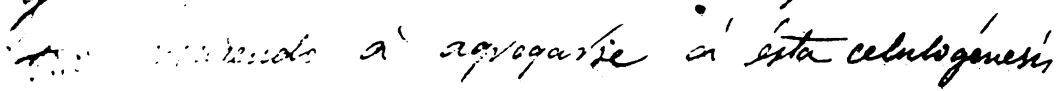


EL APRENDIZAJE HISTOLÓGICO INICIAL DE SANTIAGO RAMÓN Y CAJAL

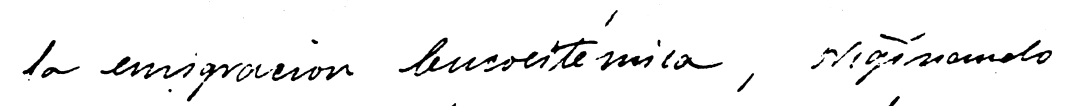

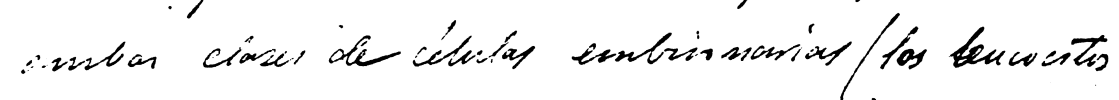
no is diterencion en in aspecto de las tóles) grow pow

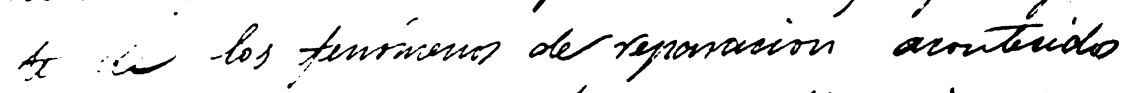
en la terizinaion de las intlamaciones.

\section{Exudados.}

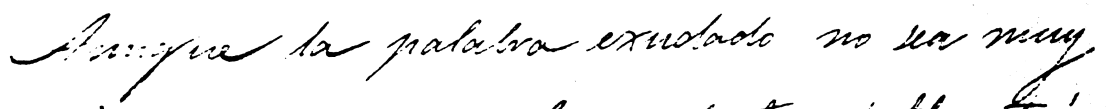

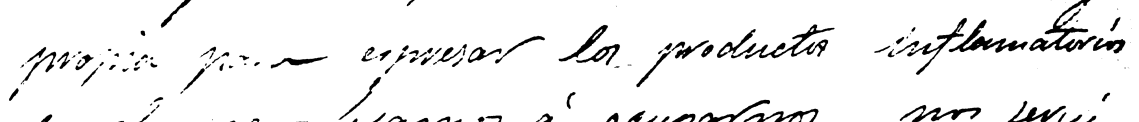

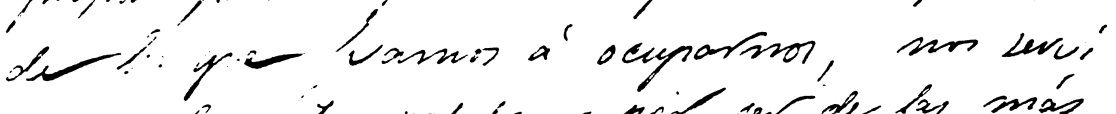
remon dee itu nalobra por jer de bas más

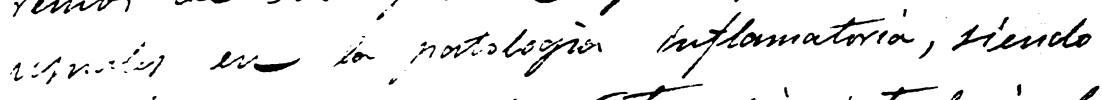

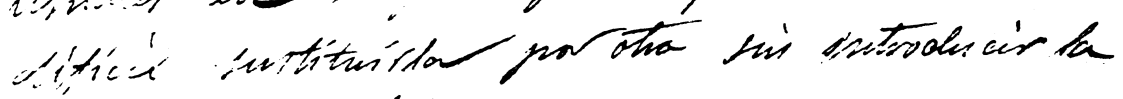
inifigis: en et lenquage.

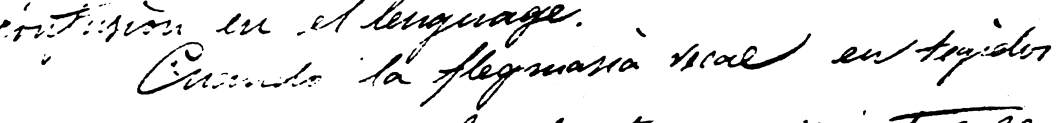
provite de rowor abundanter, y mistras ze

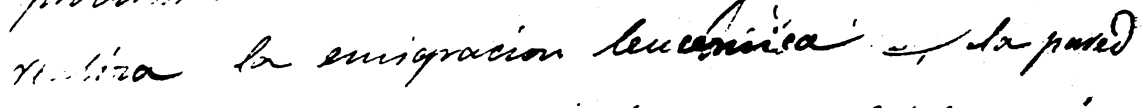

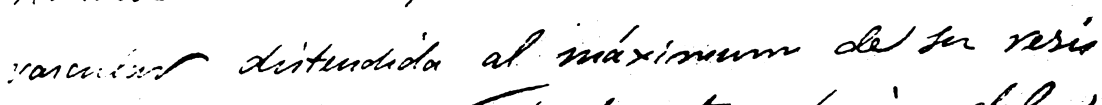

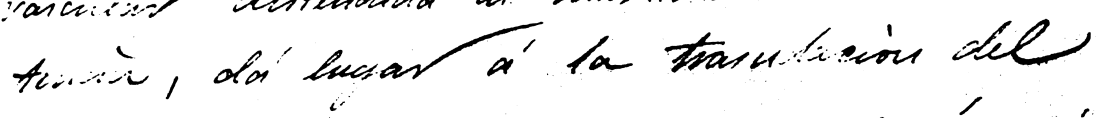

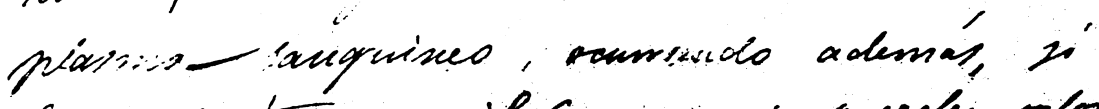
liay- intwal copilatef, gue de mesclen -lo 
ALFREDO RODRÍGUEZ QUIROGA

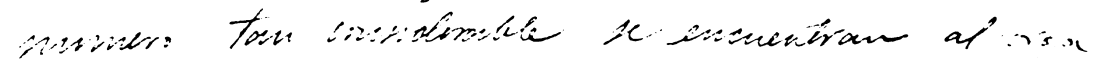

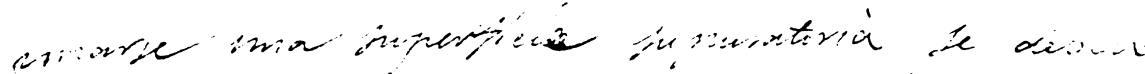

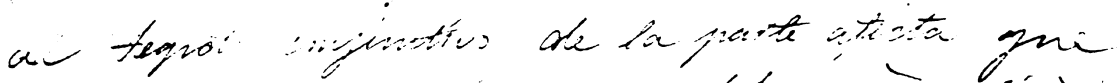

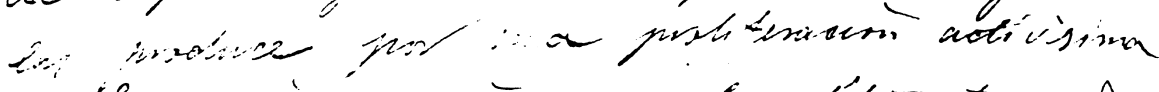

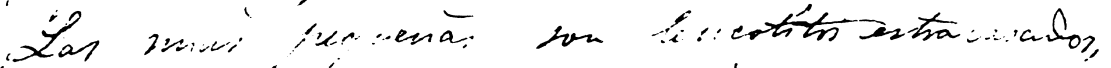

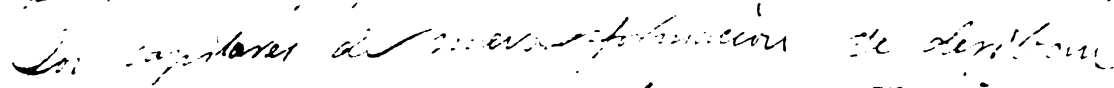

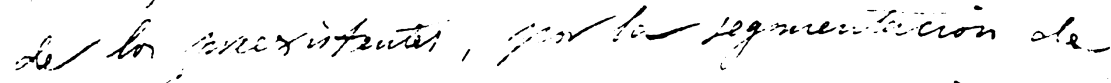

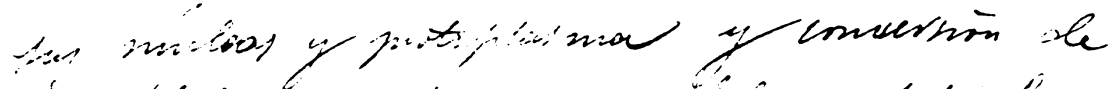

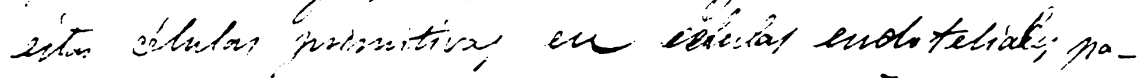

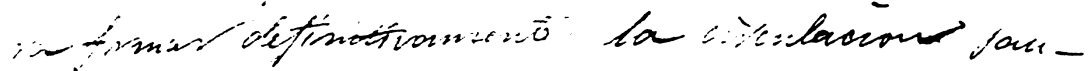

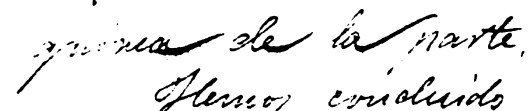

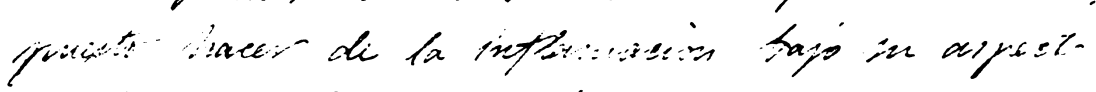

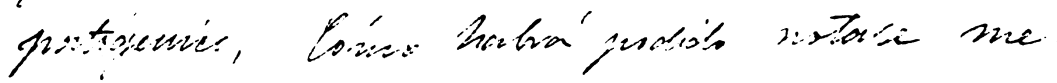

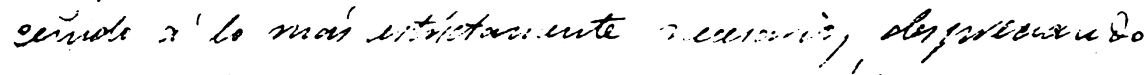

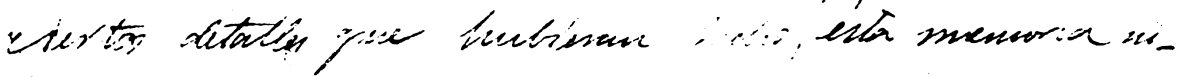
tarinsinalice.

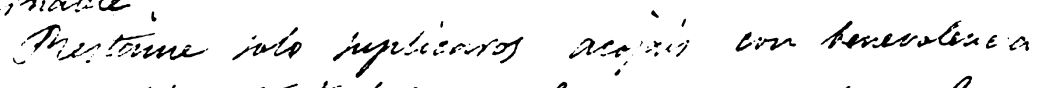

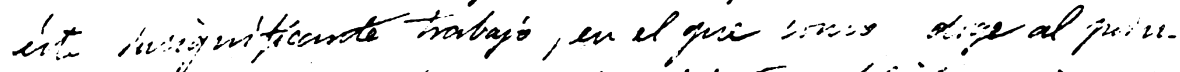

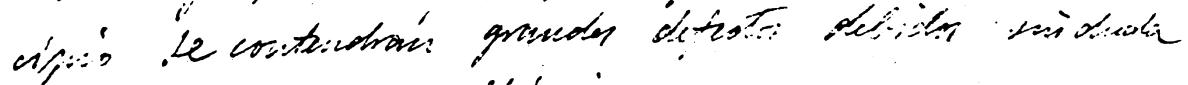

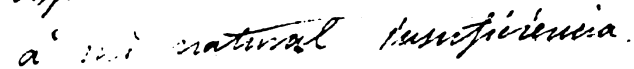
Ste'dieleo Hinchel 26 da- Eumi de MY5

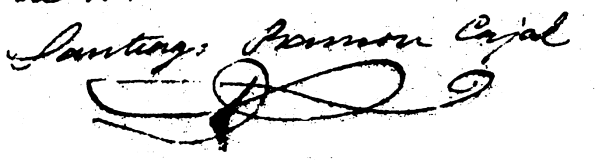


Entre las hipótesis más relevantes de este período que bien podría denominarse «conjetural», Cajal hizo referencia a las formuladas por los grandes sistemáticos, comenzando por Boerhaave para quien, de acuerdo con su sistema nosológico, en el proceso de la inflamación se producía un trastorno circulatorio consistente en un estancamiento de los vasos más angostos y un incremento en la velocidad de la sangre, de la cual resultaba una presión aumentada sobre la materia obstructiva. Stahl, por su parte, entendió la inflamación, según recogió Cajal, como «el extasis de la sangre y el esfuerzo del alma racional por desembarazarse de la causa perturbadora $\rangle^{14}$. En efecto, para Stahl —al igual que para Hoffmann-, el estasis o congestión era la «causa» material próxima de la inflamación; esto es, una dificultad de la corriente sanguínea, localizada en una determinada región del cuerpo que interpretó como una reacción protectora del organismo, es decir, del cuerpo gobernado por el anima ${ }^{15}$.

Sin embargo, según reconoció Cajal, «conforme las nociones de la anatomía patológica fueron extendiéndose, fue advirtiéndose mejor el papel que desempeñan los vasos, la sangre y los tejidos perivasculares en este proceso, naciendo tras experiencias repetidas, opiniones más en conformidad con los hechos y más próximas a la realidad» ${ }^{16}$. Destacó, entre ellas, la formulada por la denominada escuela de Viena, basada en la concepción galénica del proceso flegmásico, que concedió una importancia preeminente al exudado, como afirmó Rokitansky refiriéndose a las inflamaciones localizadas en los tejidos vasculares: «La inflamación es un proceso que comenzando en el éxtasis termina por la exudación» ${ }^{17}$, definición que, a juicio de Cajal, determinaba de manera más exacta las diferencias existentes entre la hiperemia y la inflamación, si bien seguía adoleciendo de inexactitudes dependientes principalmente del desconocimiento de las modificaciones que sobrevienen en los elementos histológicos de los tejidos inflamados. Por su parte, Jaccoud, en su Patología interna, se pronunció al respecto opinando, acertadamente según Cajal, del siguiente modo: «Hay en esta fórmula - refiriéndose a la de Rokitansky - un error y una laguna. El error es la preformación del exudado; la laguna la exclusión de los tejidos privados de vasos rojos. La definición implica una formación intravascular del líquido que trasuda en el tejido inflamado; y a ésto es lo que yo llamo preformación del exudado» ${ }^{18}$.

Las ideas hipotéticas presentadas sobre la génesis del proceso inflamatorio que caracterizaron a este período histórico, dieron paso finalmente a las sugeridas por la

\footnotetext{
14 RAMÓN Y CAJAL (1877).

15 StAHL, G. E. (1831-1833), Theoria medica vera, physiologiam et pathologiam tanquam doctrinae medicae partes vere contemplativas e naturae et artis veris fundamentis, intaminata ratione et inconcussa experienta sistens, Lipsiae, sumptibus Leopoldi Vossii.

16 RAMÓN Y CAJAL (1877).

17 GARCÍA Y SOLÁ (1874); RoKITANSKY, K. F. VON. (1842-1846), Handbuch der pathologischen Anatomie, Wien, Braunmüller \& Seidel.

18 JACCOUd, F. S. (1872-1877), Tratado de Patología interna, Madrid, Carlos Bailly-Baillière; Ramón y Cajal (1877).
} 
investigación experimental y por el exámen directo de los tejidos inflamados. Fueron los trabajos emprendidos en 1800 por los patólogos ingleses Wilson y Boraston los que marcaron el inicio de este nuevo período en el estudio de las flegmasías locales ${ }^{19}$, formulando una doctrina que, según señaló Cajal, «ha sido, después de la fórmula de la escuela de Viena, una de la que más importancia ha tenido antes de la aparición de los trabajos de Virchow ${ }^{20}$. Los experimentos de estos autores, realizados sobre el mesenterio de conejo y la membrana interdigital de la rana, cuyas partes, siendo transparentes, facilitaban la exploración microscópica de los vasos colocados en su espesor, y tras someterlos a irritación química o mecánica, permitieron observar: « $1^{\circ}$ Estrechamiento de los vasos en la porción irritada; estrechamiento que dura de 10 á 12 minutos. $2^{\circ}$ Dilatación consecutiva de los mismos capilares, los que adquieren un tamaño superior al que normalmente tienen. $3^{\circ}$ Retardo y cesación del círculo. $4^{\circ}$ Obstrucción de la cavidad vascular por el apilamiento de los hematíes, ocurriendo después los trastornos propios de las partes sometidas a los trabajos flegmásicos $»^{21}$.

Dichas conclusiones fueron plenamente sancionadas experimentalmente con posterioridad por otros autores: Thomsom (1813), Hastings (1820), Burdach (1824) y Lebert (1845), entre los más significativos; pero, a pesar de indicar ciertas modificaciones vasculares, dicha teoría no expresó el fenómeno más notable de cuantos — según se consideraba y Cohnheim evidenció posteriormente - acontecían en la inflamación de los tejidos vascularizados: la salida de los glóbulos blancos y su emigración a través de los elementos histológicos perivasculares. Además, Cajal consideró esta doctrina «incompleta, puesto que - según afirmó en su exposición - son eliminadas del proceso flegmásico todas aquellas partes que, como la córnea, cartílago, tendones, epiteliums, carecen de vasos rojos, no dándose tampoco en ella participación á la célula del tejido inflamado en la génesis de este proceso, cuando no cabe duda que en los tejidos anteriormente indicados, se inicia principalmente por una producción considerable de células embrionarias á expensas de las preexistentes, siendo casi la única metamorfosis sobrevenida durante toda la afección $»^{22}$.

Para refutar sólidamente la teoría de Wilson y Boraston tuvo que surgir la figura de Virchow, quien se ocupó de realizar una acerba crítica a dicha doctrina. No obstante, antes de abordar con detalle la nueva teoría, Cajal hizo referencia a las opiniones vertidas por Marschall-Hall acerca de la inflamación ${ }^{23}$, para quien «todas sus causas producen desde un principio sobre la superficie interna de los capilares una

19 WiLsOn, Ph. (1801), A treatise on febrile diseases, vol. III, pp. 45, 49.

RAMÓN Y CAJAL (1877).

1 Ibidem.

2 Ibidem.

23 GRAVES, R. J. (1872). Lecciones de Clínica médica (...) precedidas de una introducción del profesor Trousseau, Madrid, Carlos Bailly-Baillière. 
modificación física que lleva consigo la adherencia de los glóbulos sanguíneos a las paredes del vaso y, por consiguiente, su estancación. A medida que la inflamación crece, este éstasis aumenta y se hace más extenso, y él es el que parece constituir el carácter esencial de la lesión» ${ }^{24}$. Según Hall, pues, el primer grado lo caracterizaría la adherencia de los glóbulos de la sangre a la pared interna de los capilares, la cual disminuye considerablemente su calibre, amenazando poco a poco con llegar a la obstrucción; de este modo quedaba establecido el éstasis sanguíneo que Hall consideró como el carácter esencial de la inflamación.

Graves se encargó, como así refiere Cajal, de refutar la anterior doctrina, afirmando que «la inflamación depende de la actividad vital de los capilares, que obrando independientemente del corazón y aun de las influencias nerviosas generales, producen una hiperemia activa de orígen capilar, tras la que sobrevienen todos los fenómenos secundarios de la inflamación ${ }^{25}$. Esta teoría fue criticada por el propio Cajal al considerarla claramente en contradicción con las nociones anatómicas del momento, pues, según él, «sólo en las pequeñas arterias se encuentran fibrocélulas, y para que se efectúe la dilatación vascular más que la contracción de la fibra muscular orgánica, se requiere su paralización y, por consiguiente, desaparece esa actividad inicial a la que Graves da tanta importancia» ${ }^{26}$.

La publicación en 1858 de Die cellularpathologie de Virchow vino, en definitiva, a situar en las células, a las cuales se les concedía una vitalidad y actividad propias, el punto de partida de los fenómenos íntimos de la inflamación ${ }^{27}$. Según refleja Cajal, ésta fue considerada por el profesor de Berlín como «una perturbación de la nutrición que consiste en un cambio de materiales que tienen lugar entre la sangre y la célula que se modifica bajo el punto de vista de la nutrición, de la función y de la formación ${ }^{28}$. En esencia, para Virchow el fenómeno principal del proceso flegmásico lo constituía la irritación de la célula que provocaba una alteración de la trama orgánica, quedando relegados así a un segundo término los trastornos circulatorios del sitio afecto, es decir, la hiperemia de los vasos.

Jaccoud, por su parte, definió más claramente este proceso considerándolo «un desorden de nutrición provocado en el tejido vivo por una impresión anormal llamada irritante, hallándose constituido por la exageración temporal de la actividad nutritiva en aquella parte del organismo sometida a la irritación ${ }^{29}$.

Dicha hipótesis no se encontraba, empero, exenta de objeciones que Cajal expuso sistemáticamente pues, según esta definición, «el proceso hiperplásico fisiológico, el

24 RAMÓN Y CAJAL (1877).

25 GRAVES (1872); Ramón y Cajal (1877).

26 RAMÓN Y CAJAL (1877).

27 VIRCHOW (1858), (1868).

28 RAMÓN Y CAJAL (1877).

29 GARCíA SOLÁ (1874); Jaccoud (1872-1877). 
del crecimiento, se parece muy mucho al inflamatorio, habiendo casi identidad entre las neoplasias hiperplásicas que sobrevienen sin inflamación, y la inflamación misma; pues en todas hay exageración temporal de la actividad nutritiva de la parte irritada y en todos estos estados hay desórdenes más ó menos graduados en la nutrición de los órganos afectos, no pudiendo establecerse, según ese modo de ver las cosas, diferencias bien determinadas y exactas entre la inflamación y todas las diversas alteraciones de la nutrición» ${ }^{30}$. Asimismo, según reconoció Cajal la teoría propugnada por Virchow era, desde el punto de vista histológico, reprobable por lo que se refería a los tejidos vasculares, considerando que éstos presentaban, como fenómeno característico de su flegmasía, una extravasación de glóbulos blancos de fácil comprobación; y la proliferación celular del tejido donde se asentaba esta lesión, o no se observaba o se presentaba muy rara vez, constituyendo, por tanto, uno de los fenómenos accesorios y sucedáneos de la evolución patológica.

Llegado a este punto, Cajal abordó en su discurso la teoría que en aquel momento, y en detrimento de la formulada por Virchow, se encontraba admitida de modo más general pues explicaba de una manera satisfactoria casi todas las formas clínicas del proceso inflamatorio. Se trataba, en suma, de los fenómenos que Addison (1849) y Zimmerman (1852) habían observado en sus experiencias, comprobando «que la inflamación se acompañaba de la salida de los glóbulos blancos a través de las paredes vasculares intactas $»^{31}$, fenómeno que, más tarde, Cohnheim $(1867,1873)$ reprodujo y confirmó con investigaciones originales ${ }^{32}$. Las conclusiones más importantes de estos experimentos fueron recogidas por Cajal en su memoria donde anotó: « $1^{\circ}$. Aplicando un irritante sobre una parte de un animal y sometido a la observación microscópica, se observa en la parte irritada como fenómeno inicial una dilatación general de las venas y arterias más pequeñas. $2^{\circ}$. Lentitud considerable del círculo. $3^{\circ}$. Tendencia de los glóbulos blancos a adherirse a la superficie interna de los vasillos venosos principalmente, aunque este fenómeno también se observa en los arteriales. $4^{\circ}$. Salida de los leucocitos a través de las paredes vasculares por unos pequeños orificios, visibles con el microscopio por el método de la argentación, llamados stóma-

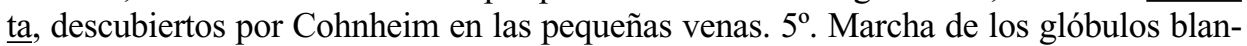
cos entre el tejido perivascular por medio de los movimientos amiboides de que están espontáneamente dotados, y agrupamiento de los mismos para formar focos, dando lugar a lo que se llama supuración en patología quirúrgica. $6^{\circ}$. Salida del plasma sanguíneo a través de las túnicas vasculares y formación del exudado con los elementos líquidos y algunos sólidos de la sangre» ${ }^{33}$. En primera instancia, Cajal juzgó 
exactas las observaciones efectuadas por Cohnheim en cuanto que hacían referencia a los tejidos vasculares, si bien cuando se trataba de tejidos carentes de vascularización las consideró un tanto exclusivas.

Según lo anteriormente expuesto, la inflamación era entendida como una lesión constituida, en los tejidos vasculares, por la extravasación de los leucocitos; y en los tejidos carentes de vasos, por una proliferación celular del tejido afecto, a la cual se uniría la penetración en la zona inflamada de algunos glóbulos blancos originados en los vasos más próximos. El proceso variaba, pues, dependiendo de la clase de tejido que se considerase; por tal motivo, Cajal se ocupó a continuación de la génesis en cada uno de aquellos dos casos.

\section{GÉNESIS. PRODUCTOS DE LA INFLAMACIÓN Y TERMINACIONES}

En efecto, Cajal consagró la segunda parte de su discurso a enunciar y describir sintéticamente los principales fenómenos de la flogosis, «según las más recientes experiencias». De este modo, expuso en primer lugar la génesis de la inflamación en los tejidos no vasculares (córnea, cartílago, tendón y epiteliums), según era entendida tras las observaciones extraídas por Cornil y Ranvier en sus experimentos efectuados en cartílagos costales de animales vivos ${ }^{34}$. Cajal, pues, admitió que, en estos tejidos, «el proceso flegmásico se inicia con un fenómeno de proliferación celular seguido de la hiperemia de los tejidos próximos al punto lesionado; hiperemia que, según Virchow es consecutiva a la irritación nutritiva y formativa a que está sometida la célula, para cuyas funciones precisa un gasto considerable de materiales $\rangle^{35}$.

En cambio, si la parte inflamada «contiene vasos rojos», la evolución patológica del proceso flegmásico presentaría un desarrollo muy diferente que Cajal caracterizó, apoyándose en las investigaciones de Cohnheim, por los siguientes fenómenos: «Comienzo por una hiperemia activa, en la que resalta sobremanera la dilatación vascular arterio-venosa, á la que sigue rápidamente el retardo del circulo sanguíneo, debido á la mayor amplitud de los vasos dilatados. Seguidamente y debido á la circulación periférica de los leucocitos, dotados de movimientos de rotación y traslación, y la subsiguiente acumulación y adhesión á la túnica interna de los vasos, sobreviene la salida, en virtud de sus movimientos amiboides, de los glóbulos blancos á través de las paredes vasculares intactas, extravasación que, por regla general, sólo podía apreciarse en las pequeñas venas y que se realizaba por unos orificios visibles al microscopio: los stómata descritos por Cohnheim» ${ }^{36}$.

\footnotetext{
34 CORNIL, A. V.; RANVIER, L. A. (1869), Manuel d'histologie pathologique, París, G. Baillière.

35 RAMÓN Y CAJAL (1877); VIRCHOW (1868).

36 COHNHEIM (1867); RAMÓN Y CAJAL (1877). 
Permanecía, sin embargo, aún por dilucidar la cuestión de la hipergenesia celular, considerada por Virchow, tanto en los tejidos vasculares como en los «blancos», el punto de partida de la afección flegmásica y según la cual las células del tejido donde se desarrollaba la flegmasía, experimentarían una irritación generativa que las haría proliferar en multitud de células embrionarias, las cuales, uniéndose a los leucocitos, constituirían la mayor parte de lo que anteriormente se denominaba «tejido inflamatorio» ${ }^{37}$. Para la mayoría de los autores, por el contrario, «los elementos histológicos autóctonos ó fijos del órgano inflamado, permanecerían en general sin la menor alteración en medio de las mencionadas perturbaciones $»^{38}$.

El estudio de la génesis inflamatoria desarrollado por Cajal le permitió a continuación exponer el proceso de formación y constitución de los distintos productos originados por este fenómeno que, de modo impropio, como él mismo reconoció, denominó como exudados. Ciertamente, este término, generalmente empleado, era inexacto puesto que parecía indicar que este líquido saldría de los vasos con todas sus cualidades, como admitía la escuela de Viena; pero ya había quedado aclarado que las alteraciones de la sangre, en vez de ser primitivas, eran constantemente consecutivas, y que el aumento de fibrina era el resultado y no la causa del proceso inflamatorio. Hunter fue el primero que concedió importancia al exudado inflamatorio, al que dió el nombre de linfa coagulable, creyendo que se organizaba directamente transformándose en tejido de cicatriz.

En definitiva, bajo la denominación de exudado se agrupaban, según hacía constar García Solá, «todas las sustancias que accidentalmente se encuentran en la trama de los órganos inflamados, y que deben su existencia al trabajo morboso de que éstos son asiento» ${ }^{39}$. Así, cuando la flegmasía acontecía en tejidos profusamente vascularizados, el «producto inflamatorio matriz» - del cual derivarían todos los demás exudados- estaría formado por los leucocitos, los hematíes y el plasma extravasado; mientras que, en los tejidos no vasculares, estaría compuesto por las células embrionarias derivadas o engendradas por la proliferación de los elementos celulares preexistentes. Cajal distinguió, siempre de acuerdo con la terminología empleada por García Solá, los siguientes productos de exudación: seroso, mucoso, fibrinoso, crupal (según la denominación de los patólogos alemanes) y purulento $^{40}$, diferenciados principalmente por su composición y, más exactamente, por la proporción relativa de fibrina y serosidad que presentaban. Particular interés evidenció Cajal por el último de los productos pues, según afirmó, «una de las consecuencias más comunes de la

37 VIRCHOW (1868).

8 RAMÓN Y CAJAL (1877).

GARCÍA SOLÁ (1874).

40 GARCÍA SOLÁ (1874); RAMÓN Y CAJAL (1877). 
inflamación es la producción de pus» ${ }^{41}$, siendo, además, la pyogenia una de las cuestiones más debatidas en aquel momento por los patólogos.

Efectivamente, respecto al mecanismo de producción de pus en un organismo inflamado se debatían distintas hipótesis entre las que, para los tejidos no vasculares, gozaba de mayor grado de aceptación la denominada «teoría celular», propugnada por Küss y Virchow ${ }^{42}$, que admitía la procedencia directa de los leucocitos del pus de las células de la región inflamada por hipergénesis o proliferación. En opinión de Cajal, en este tipo de tejidos: «la supuración está constituída simplemente por las células jóvenes producidas por la segmentación de los elementos del órgano inflamado, no interviniendo, sino indirectamente el aflujo sanguíneo en los capilares próximos» ${ }^{43}$. Del mismo modo, en los tejidos vasculares, prevalecía la hipótesis denominada de la «emigración», que, iniciada por Ang Waller en 1846 en Londres y desarrollada y sustentada con posterioridad principalmente por Cohnheim ${ }^{44}$, consideraba, en esencia, a los glóbulos de pus como simples leucocitos sanguíneos extravasados; teoría que Cajal describió como sigue: «Las células blancas que se extravasan cuando son en número considerable se coleccionan, ejercen una presión excéntrica en las mallas del tejido conjuntivo, y se labran una cavidad á que se ha dado el nombre de foco ó abceso supuratorio. Si las células blancas se esparcen entre las trabéculas del tejido conectivo, sin formar una agrupación circunscrita, fórmase la infiltración supuratoria. Al mismo tiempo, y paralelamente á estas transformaciones exuda la pared de los capilares cierta cantidad de plasma que reuniéndose con los leucocitos extravasados forma el pus; ocurriendo entonces un nuevo fenómeno que concluye de dar al leucocito todas las cualidades de célula purulenta: es la segmentación del núcleo en dos ó más, modificación que lo hace también análogo con la célula embrionaria del tejido conectivo» ${ }^{45}$.

Finalmente, Cajal concluyó su exposición ocupándose de las distintas terminaciones del proceso patológico flegmásico, que se consideraba bien podía acontecer por resolución, fenómeno por el cual el tejido inflamado volvería a sus propiedades normales y pleno ejercicio funcional; organización, cuando la tendencia de la inflamación se dirigía a formar nuevos tejidos, lo cual sucedería mediante «los procedimientos comunes de que se vale la naturaleza», y, por último, por la directa y progresiva «degeneración de los elementos celulares» ${ }^{46}$.

\footnotetext{
41 RAMÓN Y CAJAL (1877).

42 KÜSS, E. (1846). De la vascularité et de l'inflamation, Estrasburgo; (1876). Curso de Fisiología, según las lecciones del profesor (...) publicado por el Dr. Mathias Duval (1872), Madrid, Impr. de Lázaro Maroto y Roldán; Virchow (1868).

43 RAMÓN Y CAJAL (1877).

44 COHNHEIM (1867).

45 RAMÓN Y CAJAL (1877).

46 Ibidem.
} 


\section{LOS PRIMEROS ENSAYOS MICROGRÁFICOS. LA PUBLICACIÓN DE LA PRIMERA MO- NOGRAFÍA CIENTÍFICA DE CAJAL}

Durante la década comprendida entre mediados de junio de 1877, tras obtener el grado de doctor, y finales de 1887, Cajal adquirió la formación histológica que constituyó el inicio de su genial obra sobre la estructura del sistema nervioso. Decisiva resultó ser, en este sentido, como ya hemos apuntado, la relación que, con motivo de los ejercicios de doctorado, entabló Cajal con Aureliano Maestre de San Juan, en cuyo laboratorio histológico de la Facultad de Medicina pudo ver por primera vez preparaciones micrográficas y a propósito de lo cual, el propio Cajal refirió en sus Recuerdos: «Sugestionado por algunas bellas preparaciones micrográficas que el doctor Maestre de San Juan y sus ayudantes (el doctor López García entre otros) tuvieron la bondad de mostrarme, y deseoso por otra parte de aprender lo mejor posible la anatomía general, complemento indispensable de la descriptiva, resolví, a mi regreso a Zaragoza, crearme un laboratorio micrográfico» ${ }^{47}$. Influyó además notablemente en esa decisión el hecho de que en la Facultad de Medicina de esta última ciudad, como más adelante recordó Cajal en su autobiografía: «Sólo en el Laboratorio de Fisiología existía un microscopio bastante bueno. Con este veterano instrumento, y gracias a la buena amistad con que me distinguía el doctor Borao, por entonces ayudante de Fisiología, admiré por primera vez el sorprendente espectáculo de la circulación de la sangre. Tan sugerente demostración contribuyó sobremanera a desarrollar en mí la afición a los estudios micrográficos» ${ }^{48}$.

De este modo, la instalación de su laboratorio particular obligó a Cajal a invertir todas sus reservas económicas procedentes de su asignación como médico militar, adquiriendo un microscopio «Verick», un microtomo y otros instrumentos de técnica histológica. Los principales textos que manejó en estos primeros pasos como histólogo estuvieron condicionados por su paso por la cátedra de Madrid y fueron, según él mismo refiere: «Las versiones francesas de la Anatomía general de Henle ${ }^{49}$, y del Tratado clásico de Histología e Histoquimia de Frey ${ }^{50}$. El Van Kempen ${ }^{51}$ y el Robin $^{52}$. Para los trabajos clásicos pude consultar el Microscopio en Medicina de Bea-

47 RAMÓN Y CAJAL (1923).

48 RAMÓN Y CAJAL (1923).

49 Henle, J. (1843), Traité d'anatomie générale; ou, Histoires des tissus et de la composition chimique du corps humain, París, Baillière.

50 FREY, J. F. (1870), Traité d'histologie et d'histochimie, trad. par Spillmann, París.

51 VAn KeMPEN, E. M. (1863), Manual de Anatomía general (...), traducido por D. Rafael Martínez y Molina, Madrid, Carlos Bailly-Baillière.

52 RoBIN, Ch. (1866), Leçons sur l'inflamation, París; (1867), Leçons sur les vaisseaux capillaires et l'inflamation, París; (1868), Anatomie microscopique des éléments anatomiques, des épithélimus; anatomie et physiologie comparées, París, G. Baillière. 
$1 \mathrm{e}^{53}$, su Protoplasma y vida ${ }^{54}$ y el conocido Manual técnico, de Latteux ${ }^{55}$. En cuanto a las revistas científicas: suscripción y abono de unos Archivos ingleses (The Quaterly microscopical Science) $)^{56}$, y a una revista mensual francesa, dirigida por E. Pelletan (Journal de micrografie) $^{57}$. De obras españolas disponía de la del doctor Maestre de San $\operatorname{Juan}^{58}$, muy copiosa en datos, aunque de lectura un tanto difícil» ${ }^{59}$.

$\mathrm{Su}$ trabajo en dicho laboratorio, que compatibilizó con su preparación para las oposiciones a las cátedras de anatomía de Zaragoza y Granada, se limitó entonces a «curiosear sin método y desflorar asuntos». Perseveró sobre todo en el tema de la inflamación experimental, cuestión que desarrolló en su tesis doctoral y sobre la que emprendió una serie de ensayos micrográficos que le permitieron en última instancia publicar el que fue su primer trabajo científico: Investigaciones experimentales sobre la inflamación en el mesenterio, la córnea y el cartílago ${ }^{60}$, que vió la luz en 1880 ilustrado incluso con dos grabados litográficos realizados por el propio Cajal quien corrió incluso con los gastos de los cien ejemplares editados.

Este primer testimonio de su labor científica consistió básicamente, como el propio subtítulo de la obra indica (Investigaciones experimentales sobre la génesis inflamatoria y especialmente sobre la emigración de los leucocitos) ${ }^{61}$, en la elucidación de la entonces controvertida cuestión entre los anatomopatólogos del mecanismo íntimo de la inflamación, y singularmente del problema del origen de los glóbulos de pus. Para ello, Cajal procedió a la repetición y verificación minuciosa de los anteriormente mencionados y entonces ya clásicos experimentos llevados a cabo por Cohnheim (1867) para demostrar que las llamadas «células del pus» no procedían de los tejidos en los que se localiza la inflamación, sino que eran leucocitos llegados con el torrente sanguíneo ${ }^{62}$. Cajal estudió especialmente el fenómeno de la diapédesis o extravasación capilar de los leucocitos, reproduciendo con la mayor similitud posible la técnica experimental desarrollada por Cohnheim en el mesenterio inflamado de la rana curarizada, llegando finalmente a una solución de compromiso o conclusión sincrética a medio camino entre la teoría de Cohnheim y las opiniones de los histólogos france-

\footnotetext{
53 BeaLe, L. S. (1854), The Microscope and its Application to Clinical Medicine, Londres, S. Highley.

54 BEALE, L. S. (1870), Protoplasm; or Life, force \& matter, Londres, Churchill.

55 LATTEUX, P. (1877), Manuel de technique microscopique; ou Guide pratique et résumé des connaissances indispensables à l'étude du microscope, París.

${ }_{56}$ Quaterly Journal of Microscopical Science, Londres.

57 Journal de Micrographie, París.

58 Maestre de San Juan A. (1879), Tratado elemental de Histología normal y patológica precedido de un resumen de técnica histológica, Madrid, Moya y Plaza.

59 RAMÓN Y CAJAL (1923).

60 RAMÓN Y CAJAL, S. (1880), Investigaciones experimentales sobre la inflamación en el mesenterio, la córnea y el cartílago, Zaragoza, Impr. de El Diario Católico.

61 RAMÓN Y CAJAL (1880)

62 COHNHeIM (1867). 
ses que negaban dicha emigración: «Por desgracia, estaba yo entonces harto influido por las ideas de Duval, Hayem y otros histólogos franceses (que negaban la diapédesis de los glóbulos blancos), y fui arrastrado a una solución de transacción, errónea conforme suelen ser en ciencia casi todas las opiniones diagonales» ${ }^{63}$.

Efectivamente, las observaciones efectuadas por Cajal le llevaron a afirmar, de acuerdo con Duval y Morel ${ }^{64}$, que los leucocitos no salen del torrente circulatorio en la flogosis experimental ordinaria, pudiéndose además constatar de un modo evidente que las células plasmáticas perivasculares del peritoneo expuestas a la acción irritante del aire, proliferan dando origen a verdaderos glóbulos de pus. La referida diapédesis o emigración descrita hasta en sus menores detalles por Waller y Cohnheim no sería, en la mayoría de los casos, a juicio de Cajal, otra cosa que «un error de interpretación» procedente de la coexistencia de varios fenómenos distintos e independientes, «que sin embargo se los mira como solidarios, y que son: la detención y adherencia del leucocito a la pared vascular; la presencia de células emigrantes análogas a los glóbulos blancos en las zonas perivasculares; y la disposición que muchos de estos elementos presentan, pues al situarse al nivel de contorno del vaso formando una eminencia hacia adentro y otra eminencia fuera, simulan perfectamente una extravasación ${ }^{65}$. Fenómeno que solamente le había sido posible observar en el mesenterio y que, además, tendría lugar solamente en «casos determinados y especiales y sólo de una manera accidental», no debiendo, pues, concedérsele ese excesivo valor que los partidarios de Cohnheim le atribuían.

Quedaba excluida por tanto sin ningún género de dudas la existencia de los denominados stómatas o stígmatas, habida cuenta que el propio Cohnheim en investigaciones posteriores (1873) abandonó su antigua creencia y pretendió explicar la susodicha emigración de los leucocitos apelando a una especie de reblandecimiento o modificación físico-química de la pared vascular ${ }^{66}$.

Sin embargo, según Cajal, no podía afirmarse lo mismo en el caso de los glóbulos rojos, cuya emigración -descrita perfectamente por Stricker ${ }^{67}$ «es el hecho más fácilmente observable de todos los que suceden en el curso del proceso flegmásico» ${ }^{68}$.

63 RAMÓN Y CAJAL (1923)

64 Duval M. (1876), Manual del microscopio en sus aplicaciones al diagnóstico y a la Clínica, traducido del francés por el Dr. D. Marcelino Gesta y Leceta, Madrid; Duval, M. et Straus. (1870), «Recherches expérimentales sur l'inflamation», Gazette médicale de Strasbourg; MOREL, G. M. (1864), Traité d'histologie normale et pathologique, París.

65 RAMÓN Y CAJAL (1880).

66 COHNHEIM (1873).

67 STRICKER, S. (1870), Studien aus dem Institute für experimentale Pathologie in Wien aus dem Jahre 1869, Wien, (I)

68 RAMÓN Y CAJAL (1880). 
EL APRENDIZAJE HISTOLÓGICO INICIAL DE SANTIAGO RAMÓN Y CAJAL

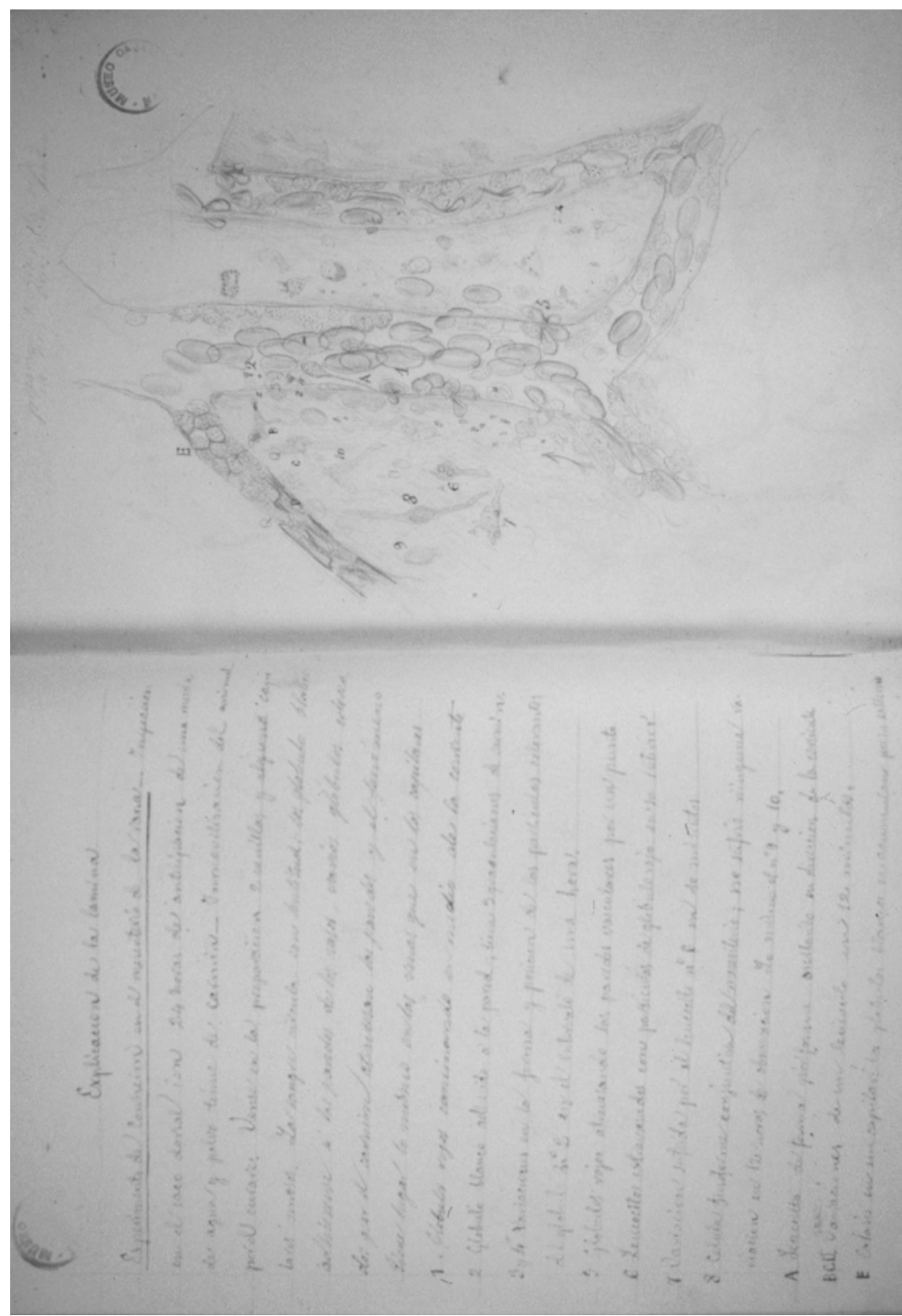

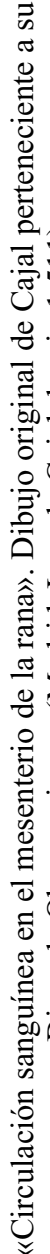

章

爱的

$\approx$.

퐁

$\cdot \frac{50}{0} \cdot \frac{\pi}{\pi}$

$\stackrel{\circ}{\overrightarrow{3}}$ 웅

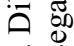

率

要

$\frac{\pi}{8} \sum^{\pi}$

윰

흥

幽

के

बे

范

颉

Asclepio-Vol. LIV-2-2002 
Asimismo, los experimentos histopatológicos efectuados por Cajal en el tejido corneal desvirtuaron notablemente las observaciones de Cohnheim al respecto, para quien la inflamación de la córnea no saldría del círculo general de las demás flegmasías, que, como en todas, estaría esencialmente constituída por la inmigración entre los elementos del órgano de corpúsculos hemáticos extravasados. Por tanto, «para que los leucocitos puedan insinuarse en los espacios del tejido corneal, es necesario que existan orificios o vías de comunicación entre las lagunas corneales y los capilares sanguíneos, por lo menos durante la flógosis. Ahora bien, en contra de esta suposición, nosotros hemos inyectado repetidas veces en el conejo las lagunas corneales con carmín amoniacal, con azul de Prusia soluble, y jamás hemos logrado llenar los vasos sanguíneos periqueráticos» ${ }^{69}$.

Se trataba, en suma, de tímidos ensayos de investigador, tildados más tarde por el propio Cajal de «bastante flojos, si bien contenían bastantes detalles nuevos acerca de las modificaciones de las células de los tejidos inflamados (córnea, cartílago, mesenterio); se señalan por primera vez la capacidad fagocítica de las plaquetas de la sangre; se estudian prolijamente las alteraciones del cemento interepitelial del peritoneo y de los capilares, etc.; pequeñas novedades que, al igual que todo lo que di a la estampa por aquellos tiempos, pasaron absolutamente inadvertidas a los sabios (...). De todos modos, con el olvido de estas menudas aportaciones, no se perdió cosa mayor $\rangle^{70}$. Ciertamente, a pesar del escaso relieve histórico de su primer trabajo científico, se advirtieron ya las dotes de excelente observador de Cajal quien, tan sólo un año más tarde y aún en pleno período de perfeccionamiento morfológico, publicó el que fue su más temprano acercamiento al sistema nervioso ${ }^{71}$. Ambos trabajos constituyen los primeros frutos de la etapa inicial de su trayectoria científica; un período que la desorientada mitificación de que ha sido objeto su figura ha conducido a minimizar, ignorando su trascendencia como verdadero punto de partida de la genial obra cajaliana.

69 Ibidem.

70 RAMÓN Y CAJAL (1923).

71 RAMÓN Y CAJAL, S. (1881), Estudios anatómicos. Observaciones microscópicas sobre las terminaciones nerviosas en los músculos voluntarios, Zaragoza, Impr. de El Diario Católico. 